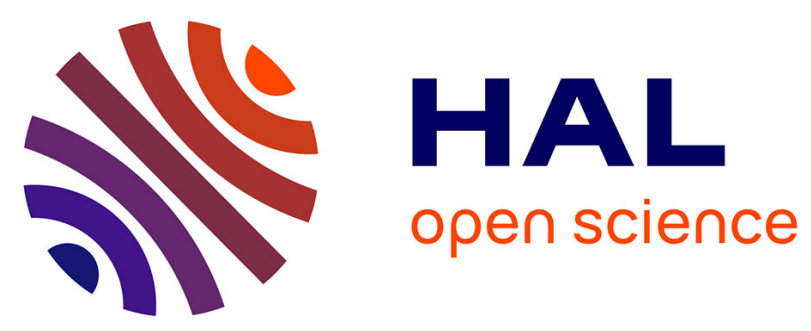

\title{
Bistability between a stationary and an oscillatory dynamo in a turbulent flow of liquid sodium
}

Mickaël Bourgoin, Michaël Berhanu, Basile Gallet, Romain Monchaux, Philippe Odier, Jean-François Pinton, Nicolas Plihon, Romain Volk, Stéphan Fauve, Nicolas Mordant, et al.

\section{To cite this version:}

Mickaël Bourgoin, Michaël Berhanu, Basile Gallet, Romain Monchaux, Philippe Odier, et al.. Bistability between a stationary and an oscillatory dynamo in a turbulent flow of liquid sodium. Journal of Fluid Mechanics, 2009, 641, pp.217-226. 10.1017/S0022112009991996 . hal-00492247

\section{HAL Id: hal-00492247 https://hal.science/hal-00492247}

Submitted on 15 Jun 2010

HAL is a multi-disciplinary open access archive for the deposit and dissemination of scientific research documents, whether they are published or not. The documents may come from teaching and research institutions in France or abroad, or from public or private research centers.
L'archive ouverte pluridisciplinaire HAL, est destinée au dépôt et à la diffusion de documents scientifiques de niveau recherche, publiés ou non, émanant des établissements d'enseignement et de recherche français ou étrangers, des laboratoires publics ou privés. 


\title{
Bistability between a stationary and an oscillatory dynamo in a turbulent flow of liquid sodium
}

\author{
M. BERHANU ${ }^{1}$, B. GALLET ${ }^{1}$, R. MONCHAUX ${ }^{2 \dagger}$, \\ M. BOURGOIN ${ }^{3} \ddagger$, PH. ODIER ${ }^{3}$, J. - F. PINTON ${ }^{3}$, N. PLIHON I $^{3}$, \\ R. VOLK ${ }^{3}$, S. FAUVE ${ }^{1}$, N. MORDANT ${ }^{1}$, F. PÉTRÉLIS ${ }^{1}$, \\ S. AUMAITRE ${ }^{2}$, A. CHIFFAUDEL ${ }^{2}$, F. DAVIAUD ${ }^{2}$, \\ B. DUBRULLE AND F. RAVELET ${ }^{2} \|$ \\ ${ }^{1}$ Laboratoire de Physique Statistique, École Normale Supérieure CNRS UMR8550, \\ 24 rue Lhomond, F-75005 Paris, France \\ ${ }^{2}$ Service de Physique de l'État Condensé, Direction des Sciences de la Matière CNRS URA 2464, \\ CEA-Saclay, F-91191 Gif-sur-Yvette, France \\ ${ }^{3}$ Laboratoire de Physique, École Normale Supérieure de Lyon, CNRS UMR5672, \\ 46 allée d'Italie, F-69364 Lyon, France
}

(Received 2 June 2009; revised 4 September 2009; accepted 4 September 2009; first published online 16 November 2009)

We report the first experimental observation of a bistable dynamo regime. A turbulent flow of liquid sodium is generated between two disks in the von Kármán geometry (VKS experiment). When one disk is kept at rest, bistability is observed between a stationary and an oscillatory magnetic field. The stationary and oscillatory branches occur in the vicinity of a codimension-two bifurcation that results from the coupling between two modes of magnetic field. We present an experimental study of the two regimes and study in detail the region of bistability that we understand in terms of dynamical system theory. Despite the very turbulent nature of the flow, the bifurcations of the magnetic field are correctly described by a low-dimensional model. In addition, the different regimes are robust; i.e. turbulent fluctuations do not drive any transition between the oscillatory and stationary states in the region of bistability.

\section{Introduction}

The magnetic field of most astrophysical objects, like planets, stars and galaxies, is generated by the dynamo effect, which is an instability that converts the kinetic energy of an electrically conducting fluid into magnetic energy. Studies on the geodynamo reveal that global rotation decreases the efficiency of convection (Chandrasekhar 1961) and therefore limits the capability of dynamo action in rapidly rotating flows. However Roberts (1978) suggested that a sufficiently strong magnetic field could create a Lorentz force in balance with the Coriolis force, which may counter the effect of strong rotation. This mechanism predicts a subcritical bifurcation either between a state of no dynamo and a state of strong field or between a weak and a strong

$\dagger$ Present address: ENSTA-UME, chemin de la Hunière, 91761 Palaiseau Cedex, France † Present address: LEGI, CNRS UMR 5519, BP53, F-38041 Grenoble, France

I Email address for correspondence: Stephan.Fauve@1ps.ens.fr

\| Present address: LEMFI, ENSAM, 151 bld de l'Hôpital, 75013 Paris, France 


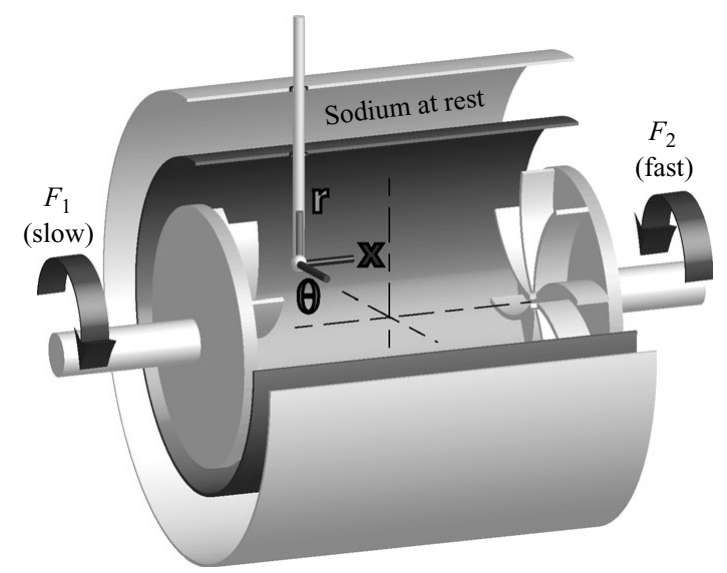

FIGURE 1. Sketch of the experiment. Two soft iron-bladed disks drive a sodium flow in a cylinder of radius $206 \mathrm{~mm}$ and length $524 \mathrm{~mm}$.

field regime. Subcritical transitions have been identified in magnetohydrodynamic numerical simulations of thermal convection in a rotating sphere (Christensen, Olson \& Glatzmaier 1999; Morin 2005; Morin \& Dormy, submitted) and of body forced flows in a sphere (Fuchs, Rädler \& Rheinhardt 2001) as well as within a periodic box (Ponty et al. 2007) and in Keplerian shear flows (Rincon, Ogilvie \& Proctor 2007). In experimental and astrophysical turbulent flows with larger Reynolds numbers $\left(R e \geqslant 10^{6}\right)$ and realistic boundary conditions, the existence of subcritical dynamo transitions remains an open question. The velocity fluctuations may smooth the transition or suppress bistability. They may also lead the system to explore different regimes successively in time.

Experimental observation of the dynamo instability in fluids has been achieved only recently in Karlsruhe (Stieglitz \& Müller 2001), Riga (Gailitis et al. 2001) and in a von Kármán swirling flow of liquid sodium (VKS experiment; Monchaux et al. 2007). A stationary magnetic field has been generated in the Karlsruhe experiment, whereas an oscillatory dynamo has been observed in Riga. In the VKS experiment, various regimes including random reversals (Berhanu et al. 2007; Monchaux et al. 2009) have been observed. All these experiments have displayed continuous primary bifurcations of the magnetic field when the flow velocity is increased. Subcriticality and bistability have not been observed so far. Here we study a turbulent flow of liquid sodium generated between two disks. By varying independently the velocity of each disk, we identify a bistable regime for the magnetic field generated by dynamo action.

The experimental set-up, sketched in figure 1, has been described in Monchaux et al. (2007). A von Kármán swirling flow is generated by two counter-rotating impellers made of soft iron disks fitted with eight blades (radius $R=154.5 \mathrm{~mm}$, distance between inner faces $371 \mathrm{~mm}$ ) in an inner copper cylinder (radius $R_{c}=206 \mathrm{~mm}$, length $524 \mathrm{~mm}$ ). We denote the rotation frequencies of the two impellers by $F_{1}$ and $F_{2}$. The fluid is liquid sodium (density $\rho \simeq 930 \mathrm{~kg} \mathrm{~m}^{-3}$, electrical conductivity $\sigma \simeq 10^{7} \mathrm{ohm}^{-1} \mathrm{~m}^{-1}$, kinematic viscosity $v \simeq 10^{-6} \mathrm{~m}^{2} \mathrm{~s}^{-1}$ ). The flow is surrounded by sodium at rest between the inner cylinder and an outer cylinder (radius $289 \mathrm{~mm}$, length $604 \mathrm{~mm}$ ). The driving motor power is $300 \mathrm{~kW}$, and cooling by an oil circulation inside the wall of the outer copper vessel allows experimental operation at constant temperature in the range $110-160^{\circ} \mathrm{C}$. The integral Reynolds numbers, defined as $R e_{i}=2 \pi R^{2} F_{i} / v(i=1,2)$, take values up 


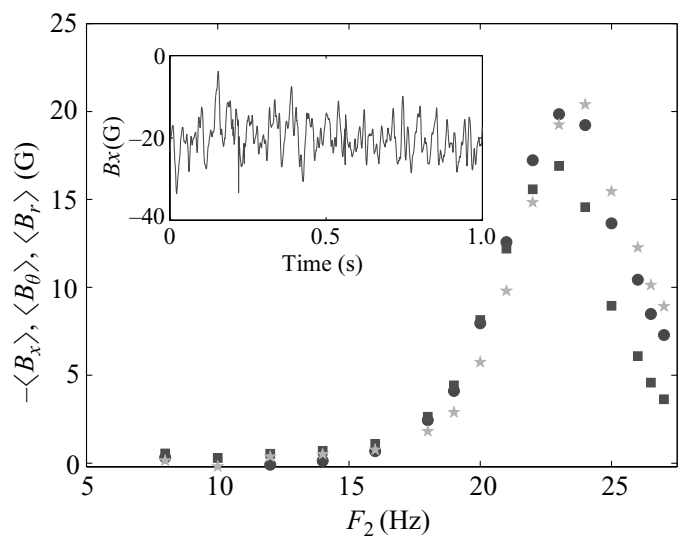

FIGURE 2. Bifurcation diagram of the stationary dynamo generated by varying $F_{2}$ with $F_{1}=0$. The symbols are as follows: $\bullet,-\left\langle B_{x}\right\rangle ; \boldsymbol{\square},\left\langle B_{\theta}\right\rangle ; \star,\left\langle B_{r}\right\rangle$. The inset shows the corresponding time series of the component $B_{x}$ of the stationary dynamo generated for $F_{1}=0$ and $F_{2}=24 \mathrm{~Hz}$.

to $5 \times 10^{6}$. Correspondingly, magnetic Reynolds numbers $R_{m i}=2 \pi \mu_{0} \sigma R^{2} F_{i}$ up to 49 at $120^{\circ} \mathrm{C}$ are reached $\left(\mu_{0}\right.$ is the magnetic permeability of vacuum). Note that the definitions of $R e$ and $R_{m}$ are different from those given in Monchaux et al. (2007) and Berhanu et al. (2007), respectively. The values of $R e$ and $R_{m}$ given here must be multiplied by $K\left(R_{c} / R\right)^{2}$, where $K=1.1$ is the efficiency of the impellers when one disk is kept at rest, to recover the value of the latter given in Berhanu et al. (2007). The only modification compared with the set-up described in Monchaux et al. (2007) is the removal of the inner copper ring in the mid-plane. Using cylindrical coordinates, we define $x$ as the coordinate along the axis of the cylinder directed from disk 1 towards disk 2. The radial coordinate is $r$ and $\theta$ is the azimuth. The coordinates of the cylinder centre are $(x=0, r=0)$. Here we consider experiments in which disk 2 rotates much faster than disk 1 . The faster disk drives global rotation in most of the fluid together with radial ejection and axial pumping, generating a one-cell flow with a turbulent shear layer close to the slow disk as illustrated in Ravelet et al. (2004). The magnetic field $\boldsymbol{B}$ is measured with the Hall probes of a three-axis gaussmeter inserted inside the fluid close to the slower disk at the position $(x=-109 \mathrm{~mm}, r=43 \mathrm{~mm})$. The experiment is operated at a sodium temperature close to $120^{\circ} \mathrm{C}$.

When the velocity of disk 2 is increased, while disk 1 is at rest, that is to say $F_{1}=0$, a magnetic field is generated for $F_{2}$ larger than $16 \mathrm{~Hz}$, i.e. $R_{m 2}$ larger than 30. This magnetic field moderately fluctuates around a mean value as shown in the time series plotted in the inset of figure 2. This dynamo is less fluctuating than the stationary dynamo obtained when the two disks exactly counter-rotate. Indeed, for $F_{1}=0$ and $F_{2}=24 \mathrm{~Hz}$ the standard deviation of the magnetic field normalized as $B_{r m s} /\left\langle B^{2}\right\rangle^{1 / 2}$ is around $20 \%$, whereas it is around $50 \%$ in the counter-rotating case for $F_{1}=F_{2}=24 \mathrm{~Hz}$. This is related to the smaller turbulent velocity fluctuations when only one disk is rotated. It has been shown indeed that the ratio of the instantaneous kinetic energy to that of the time-averaged flow is about twice as large in the counterrotating case than in the one-disk configuration (Cortet et al. 2009). Increasing the velocity of the disk by steps of $0.5 \mathrm{~Hz}$, a stationary dynamo is maintained. The time average of each component of the magnetic field $\left\langle B_{i}\right\rangle$ is displayed as a function of $F_{2}$ in figure 2. The three components of the magnetic field are of the same order of magnitude, the axial component being of the sign opposite to that of the radial 

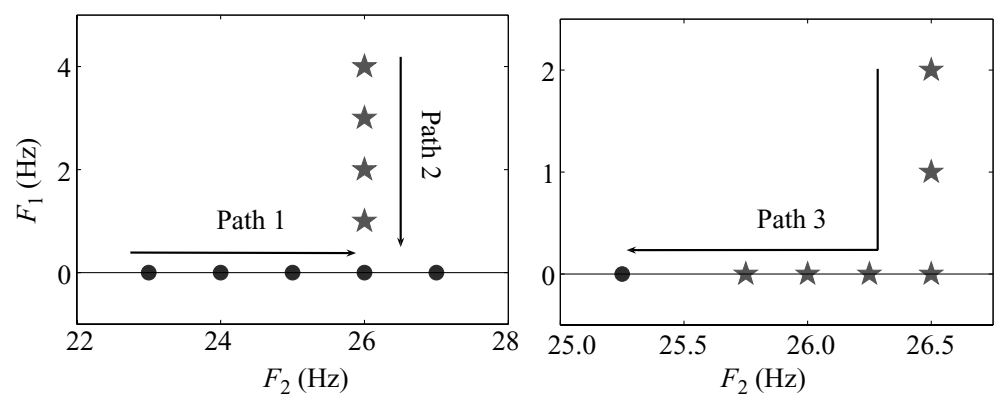

FIgURE 3. Paths followed in the parameter space $\left(F_{2}, F_{1}\right)$ : $\bullet$, stationary dynamos; $\star$, oscillatory dynamos. Note that for $(26,0)$, depending on the path, either an oscillatory or a stationary dynamo can be obtained.

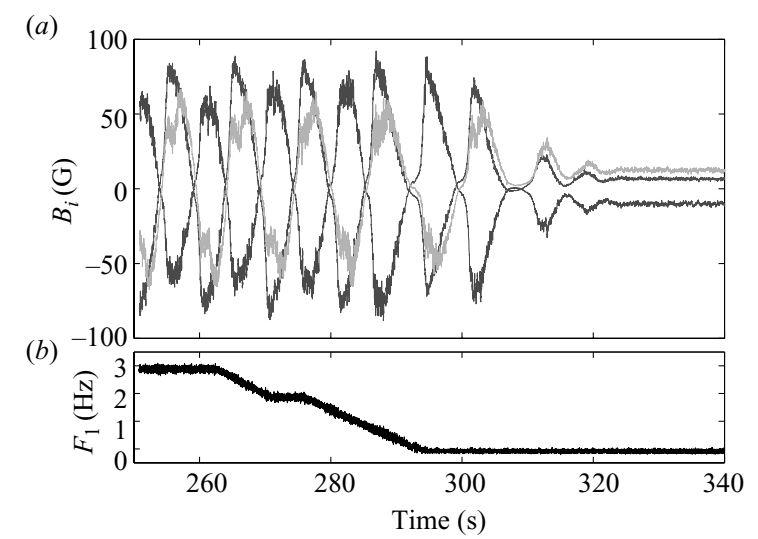

Figure 4. Time series for the oscillatory dynamo. (a) Magnetic field components as a function of time. The colours are as follows: blue, $B_{x}$; red, $B_{\theta}$; green, $B_{r} .(b)$ Velocity of the slow disk $F_{1}$ as a function of time. At $t=295 \mathrm{~s}$, the velocity of disk 1 is set to $F_{1}=0$ while $F_{2}=26 \mathrm{~Hz}$.

and azimuthal components. The sign of $\left\langle B_{x}\right\rangle\left\langle B_{\theta}\right\rangle$ is set by the one of differential rotation, as when the disks are in exact counter-rotation. Both solutions $\boldsymbol{B}$ and $-\boldsymbol{B}$ can be observed. There is an intensity difference (of the order of $5 \mathrm{G}$ ) between these branches, an effect possibly due to induction from the ambient magnetic field. The time-averaged amplitude of the magnetic field $\left\langle\boldsymbol{B}^{2}\right\rangle^{1 / 2}$ grows up to $35 \mathrm{G}$ for $F_{2} \simeq 24 \mathrm{~Hz}$ and then decreases for larger velocities.

When disk 1 slowly counter-rotates compared with disk 2, a periodic dynamo is generated. To investigate the relative stability of the stationary and oscillatory regimes, we follow three different paths (sketched in figure 3 ) in parameter space, each ending on the line $\left(F_{2}, 0\right)$ where only disk 2 rotates. Path 1 has been presented above. The two others are performed by slowing down disk 1, disk 2 being kept at a fast rotation rate (resp. at 26 and $26.5 \mathrm{~Hz}$ ). We now describe the evolution of the dynamo when following path 2. If $F_{2}=26 \mathrm{~Hz}$ and $F_{1}$ is larger than $1 \mathrm{~Hz}$, a periodic dynamo takes place, the time series of which is displayed in figure 4. At time $t=295 \mathrm{~s}$, disk 1 is stopped, and the magnetic field tends towards a stationary value after transient damped oscillations. This stationary magnetic field is similar to the field produced when following path 1 . The damped oscillations indicate that the corresponding fixed point is linearly stable with complex eigenvalues. 
(a)

(b)
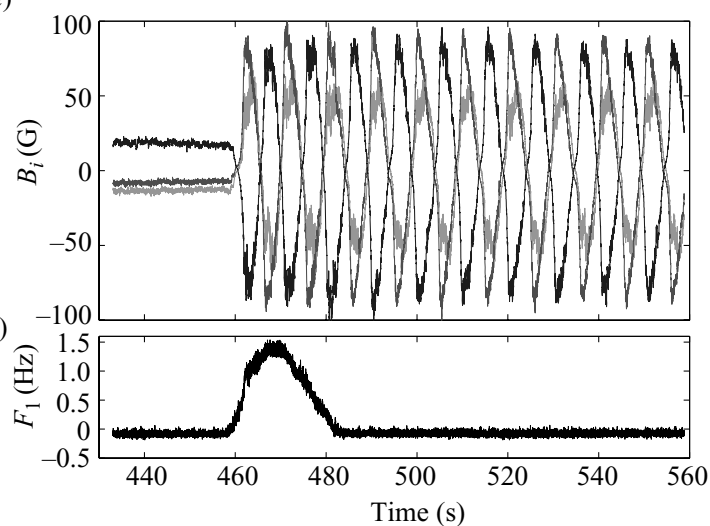

Figure 5. Time series for the oscillatory dynamo. (a) Magnetic field components as a function of time. The colours are as follows: blue, $B_{x}$; red, $B_{\theta}$; green, $B_{r} .(b)$ Velocity of the slow disk $F_{1}$ as a function of time. At $t=480 \mathrm{~s}$, the velocity of disk 1 is set to $F_{1}=0$ while $F_{2}=26.5 \mathrm{~Hz}$.

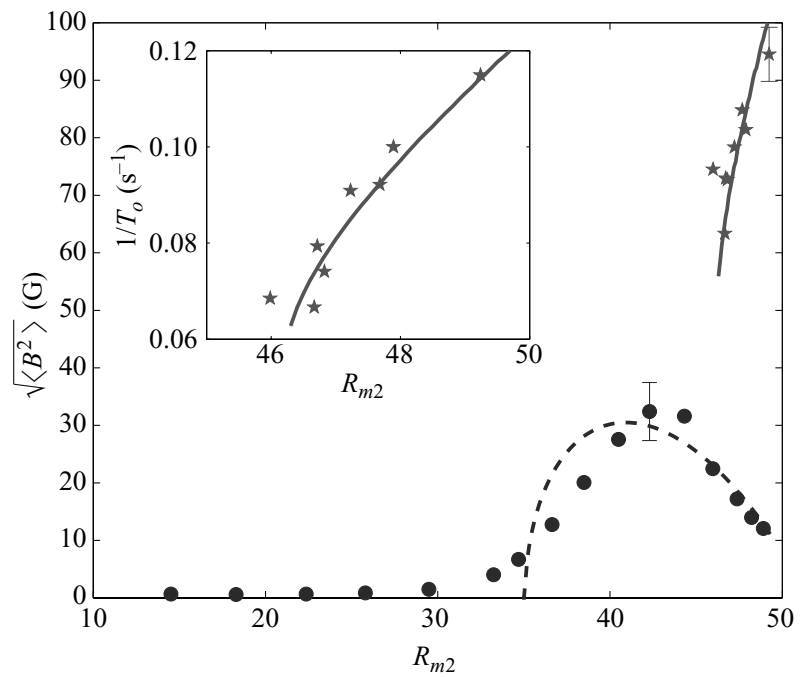

FIGURE 6. Amplitude of the magnetic field $\left\langle\boldsymbol{B}^{2}\right\rangle^{1 / 2}$ as a function of $R_{m 2}$ for $R_{m 1}=0$. The inset shows the frequency of the oscillatory dynamo. The symbols are experimental values: -, stationary dynamo; $\star$, oscillatory dynamo. The length of the error bars is obtained from the dispersion of the measurements performed for the same parameter values. The lines are obtained from the solutions of the dynamical system defined by (1): - - , oscillatory solution; --- , stationary solution.

To follow path 3 , we start with $F_{2}=26.5 \mathrm{~Hz}$ and $F_{1} \geqslant 1 \mathrm{~Hz}$. The dynamo is then periodic. When stopping disk 1 , it is possible to maintain a periodic dynamo as can be seen in figure 5 . We then decrease $F_{2}$, and the system remains in periodic dynamo regime for $F_{2}$ down to $25.75 \mathrm{~Hz}$. A stationary dynamo is finally recovered for $F_{2}=25.25 \mathrm{~Hz}$. The period of the oscillatory dynamo roughly increases from 9 to $15 \mathrm{~s}$ when $F_{2}$ is varied from 27 to $25.5 \mathrm{~Hz}$. The frequency does not vanish for the smallest velocity at which the oscillations can be maintained, as can be seen in the inset of figure 6 . The three components of the magnetic field are non-zero and are of similar amplitude. During most of the period, the axial field is of opposite sign with respect 
to the azimuthal and radial fields, as is the case in the stationary regime. There is a small phase shift between the three components, the radial one being delayed by less than one tenth of a period. Measurements at other positions (not described here) have shown that the magnetic field oscillates with roughly the same phase within the experiment. As expected for a bistable regime, this oscillatory dynamo has also been observed when one disk is at rest if the velocity of the other disk is increased by larger steps $(3 \mathrm{~Hz})$. However the oscillatory branch is more easily observed when the flow is first driven with both disks rotating.

From the former paths in the parameter space, we can state that two different dynamo regimes are observed for the same values of the control parameters. This proves the existence of two locally stable dynamo branches. We measure that the domain of bistability extends from 25.5 to $27 \mathrm{~Hz}$ in $F_{2}$. Driving one disk at $27 \mathrm{~Hz}$, the other one being at rest, requires all the available power for one disk, roughly $150 \mathrm{~kW}$. This power limitation does not allow us to reach higher frequencies, but we expect the interval of bistability to be wider. A bifurcation curve based on the norm of the magnetic field is plotted in figure 6. The amplitudes of the two branches strongly differ. The oscillatory dynamo generates a field with energy density roughly 10 times larger than that of the stationary dynamo. However, within measurement accuracy, there is no noticeable effect on the power delivered by the motor (with the change being less than $3 \%$ ).

We note that for the stationary regime and during most of the period of the oscillatory one, the axial and azimuthal components of the magnetic field are of opposite signs. This is in agreement with a mechanism of magnetic field generation put forward in Pétrélis, Mordant \& Fauve (2007): the flow of the fluid radially expelled by the disk is strongly helical and generates an $\alpha$ effect localized close to the disk. This $\alpha$ effect converts the azimuthal field into the axial field. Differential rotation is responsible for the $\omega$ effect that converts the axial magnetic field into the azimuthal one. This mechanism has been studied by Laguerre et al. (2008) in the framework of mean-field magnetohydrodynamics. It has been found that the magnitude of $\alpha$ needed to obtain the dynamo is much too large. This has been confirmed by Giesecke et al. (forthcoming). The possible role of the iron impellers has been also taken into account, and it has been shown that a smaller value of $\alpha$ is needed for dynamo action (Giesecke, Stefani \& Gerbeth, forthcoming). However, kinematic calculations performed without using any $\alpha$ parameterization have shown that taking into account non-axisymmetric vortices along the blades fairly well describes the experimental results (Gissinger, forthcoming). More measurements are necessary to fully describe the structure of the field and the amplification mechanisms. Nevertheless, our explanation for the bistability (detailed in the following) confirms the fact that the same effects are at work for both the stationary and the oscillatory regime.

To identify the dynamics that leads to bistability between the two regimes we perform a phase-space study of the magnetic field. To wit we plot the radial magnetic field as a function of the azimuthal one. In figure $7(a)$, we display the evolution corresponding to different initial conditions and $F_{1}=0, F_{2}=26 \mathrm{~Hz}$. In one case the solution spirals towards a fixed point; in the other case it describes a limit cycle. We note that the fixed point, its opposite and the $B=0$ unstable solution are located inside the limit cycle. Figure 7(b) represents the evolution of the limit cycle when $F_{2}$ is varied. It is clear that when $F_{2}$ is decreased the limit cycle comes closer to $B=0$.

These results can be understood as resulting from the competition between two modes of magnetic field in the vicinity of a codimension-two bifurcationor, more precisely, of a bifurcation for a linear problem with a double zero eigenvalue. 
(a)

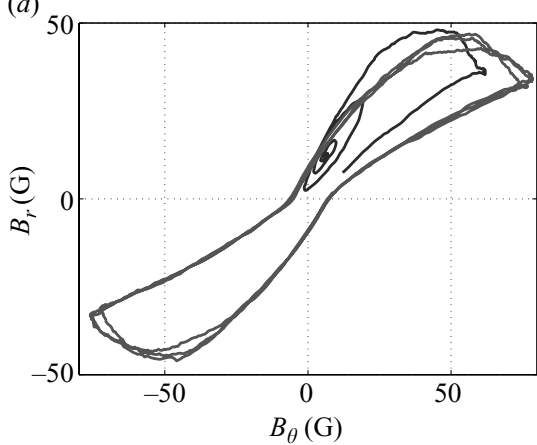

(b)

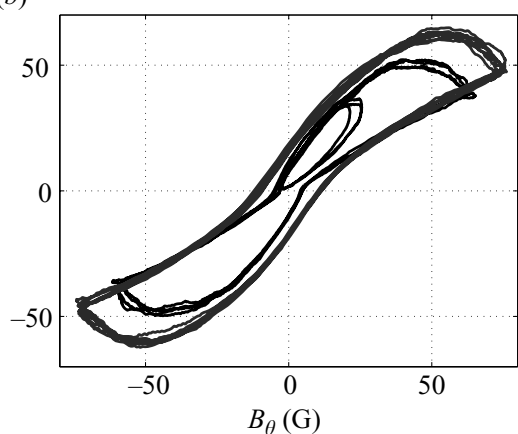

Figure 7. Plot of a cut in phase space $\left[B_{\theta}(t), B_{r}(t)\right]$ for different measurements with $F_{1}=0 \mathrm{~Hz}$. (a) $F_{2}=26 \mathrm{~Hz}$. The blue (resp. red) line is made from the time series of figure 4 (resp. figure 5) and corresponds to initial conditions attracted towards a fixed point (resp. a limit cycle). $(b)$ $F_{2}=27 \mathrm{~Hz}$ (blue) and $F_{2}=25.5 \mathrm{~Hz}$ (black). Note the initial evolution that circles around the fixed point in the upper right part of the plot before describing the limit cycle.

Following Pétrélis \& Fauve (2008), we assume that the dynamics of the magnetic field results from the competition between two magnetic modes and write the magnetic field as $\boldsymbol{B}=A_{1}(t) \boldsymbol{B}_{1}(\boldsymbol{r})+A_{2}(t) \boldsymbol{B}_{2}(\boldsymbol{r})$ where $\boldsymbol{B}_{i}$ are the spatial structures of the modes and $A_{i}$ their amplitudes. Dynamics of the magnetic field is then reduced to that of $A=A_{1}+\mathrm{i} A_{2}$. The amplitude equation for $A$ should be invariant when $A \rightarrow-A$, since the equations of magnetohydrodynamics are invariant when $\boldsymbol{B} \rightarrow-\boldsymbol{B}$. When the two modes are near criticality, we obtain up to the leading-order nonlinearities

$$
\dot{A}=\left(\delta_{1}+\mathrm{i} \delta_{2}\right) A+\left(v_{1}+\mathrm{i} v_{2}\right) \bar{A}+\beta_{1} A^{3}+\beta_{2} A^{2} \bar{A}+\beta_{3} A \bar{A}^{2}+\beta_{4} \bar{A}^{3} .
$$

Equations similar to (1) occur in many different contexts. In particular, they describe strong resonances in space (Coullet 1986) or in time (Chiffaudel \& Fauve 1987) for pattern-forming instabilities. Their bifurcation diagram has been widely studied (Arnold 1982; Gambaudo 1985).

In the general case, the coefficients $\beta_{i}$ are complex and $\delta_{1}, \delta_{2}, v_{1}$ and $v_{2}$ are real. They depend on the experimental parameters. It has been shown that when $\delta_{1}$ is large enough such that the amplitude of $A$ can be adiabatically eliminated, transitions from stationary to periodically reversing dynamos result from a saddle-node bifurcation. In addition, if turbulent fluctuations are modelled by multiplicative noise, (1) describes random reversals and bursts observed in the VKS experiment (Pétrélis \& Fauve 2008, Pétrélis et al. 2009).

When $\delta_{1}=0$ and $\delta_{2}^{2}=v_{1}^{2}+v_{2}^{2}$, (1) has a codimension-two bifurcation point with a double-zero eigenvalue and only one eigenvector; i.e. the system is close to both a stationary and a Hopf bifurcation. Indeed, the eigenvalues of the linear part of the equation are $\delta_{1} \pm \sqrt{v_{1}^{2}+v_{2}^{2}-\delta_{2}^{2}}$. Up to a rotation and a change of time scale, the linear part of (1) can be reduced to $\dot{X}=Y$ and $\dot{Y}=0$, where $X+\mathrm{i} Y=A \mathrm{e}^{-\mathrm{i}(\phi / 2+\pi / 4)}$ with $\delta_{2} \mathrm{e}^{\mathrm{i} \phi}=v_{1}+\mathrm{i} v_{2}$. The phenomenology of this codimension-two bifurcation is well documented (Guckenheimer \& Holmes 1983). At the bifurcation, the normal form can be written as $\dot{X}=Y$ and $\dot{Y}= \pm X^{2} Y-X^{3}$, up to a close-to-identity transformation and rescaling of time and amplitudes. When the two nonlinear terms of the normal form saturate the instability, the oscillation appears via a saddle-node bifurcation that creates a pair of limit cycles, a stable and an unstable one. These limit cycles encircle the three stationary solutions $\left(B=0\right.$ and the two bifurcated solutions $\left.\pm B_{s}\right)$. Once the 
limit cycles are created and as long as the stationary bifurcated solutions are stable, the system displays bistability between the stationary and the oscillatory solutions.

To illustrate this scenario, we compare the experimental results with the solution of (1) when a simple variation of the coefficients as a function of $R_{m 2}$ is prescribed. For simplicity, we keep only the nonlinear term that does not break phase invariance; i.e. we take $\beta_{1}=\beta_{3}=\beta_{4}=0$. In order to reduce further the number of fitting parameters, we note that the phase-space representation of the system is elongated along the diagonal $B_{r}=B_{\theta}$ (see figure 7). Similarly, the three components of the magnetic field remain comparable. This implies that one of the two modes, say $\boldsymbol{B}_{1}$, is larger than the second mode at the location at which the measurements are performed. Therefore the amplitude $A_{1}$ can be considered as providing a good estimation of the magnetic field at the measurement point. The remaining coefficients are then chosen such that the stationary and oscillatory instability thresholds, the oscillation amplitudes and frequencies and the maximum of the stationary solution amplitude, are correctly described. This is achieved by setting $v_{1}=0.27, v_{2}=0.52$, $\delta_{1}=0.043 R_{m 2}-1.74, \delta_{2}=1.45-0.026 R_{m 2}$ and $\beta_{2}=10^{-5}(-0.95+1.9 i)$. We obtain from (1) that the stationary solution bifurcates for $R_{m 2}=35.7$ and displays bistability with the oscillatory solution when $R_{m 2} \geqslant 46.1$. We display $\sqrt{\left\langle\boldsymbol{B}^{2}\right\rangle}$ and the period of the oscillatory solutions in figure 6 . The imperfection of the bifurcation observed in the experimental data may be attributed to the remanent magnetization of the disks. Taking into account this imperfection as well as the experimental error bars, we observe that the model describes the measurements fairly well. In other words, not only the phenomenology of the successive bifurcations but also some quantitative aspects are captured by this low-dimensional approach. This shows that the dynamical regimes observed in a fully turbulent flow result from the competition between two modes of magnetic field. Indeed, even though the Reynolds number is larger than $10^{6}$, the magnetic Reynolds number remains close to onset, and the dynamics is governed by the small number of magnetic modes contained in the central manifold.

We emphasize that the scenario described here is different from the one observed for values of $F_{i}$ closer to exact counter-rotation. In that case, the magnetic field evolves from a stationary to a periodic behaviour when one of the velocities is changed (Ravelet et al. 2008). There is no bistability, and the fixed point associated with the stationary behaviour belongs to the periodic orbit. In the vicinity of this bifurcation, turbulent fluctuations drive random reversals by excitability. In the present case, both the stationary and time periodic regimes coexist for the same parameter values. When the turbulent flow of liquid sodium is driven by the fast rotation of one disk, the magnetic field remains in one of the states, and the turbulent fluctuations are not sufficient to trigger random transitions between the two regimes on an observation time larger than hundreds of ohmic diffusive times or thousands of hydrodynamic eddy turnover times. The coexistence of a stationary and an oscillatory regime has been also observed when both disks rotate at different frequencies (for instance $F_{1}=16.75 \mathrm{~Hz}, F_{2}=25.25 \mathrm{~Hz}$ ). However, in that case random transitions between the stationary and the oscillatory regime are observed without changing the control parameters (see figure 3.69 in Berhanu 2008). This can be due either to the larger intensity of turbulent fluctuations when the two impellers are counter-rotating at comparable frequencies or to the proximity of the fixed point and the limit cycle in phase space. In any case, we stress that the low-dimensional dynamics of the magnetic field is very robust. These features can be understood as resulting from the coupling between two modes of magnetic field close to their onset of instability. Far from 
the codimension-two point of the amplitude equation (1), we have a transition from a stationary state to a time-periodic one through a saddle-node bifurcation. In the vicinity of the codimension-two point, the bistable behaviour is observed.

Finally, we emphasize that the dynamo regimes observed in the VKS experiment strongly differ from the ones computed using the mean flow alone. It is necessary to take into account non-axisymmetric fluctuations of the flow, and these fluctuations are turbulent. The validity of the low-dimensional description of the dynamics of the magnetic field relies the small number of magnetic modes competing in the vicinity of the dynamo threshold.

We are indebted to M. Moulin, C. Gasquet, J.-B. Luciani, A. Skiara, D. Courtiade, J.-F. Point, P. Metz and V. Padilla for their technical assistance at various stages of the experiment. This work is supported by the following French institutions: Direction des Sciences de la Matière and Direction de l'Energie Nucléaire of CEA, Ministère de la Recherche, and Centre National de Recherche Scientifique (ANR 08-0039-03). The experiment was operated at CEA/Cadarache DEN/DTN.

\section{REFERENCES}

ARnold, V. 1982 Geometrical Methods in the Theory of Ordinary Differential Equations. Springer.

Berhanu, M. 2008. Magnétohydrodynamique turbulente dans les métaux liquides. PhD thesis, Université Pierre et Marie Curie, Paris, p. 105.

Berhanu, M., Monchaux, R., Fauve, S., Mordant, N., Pétrélis, F., Chiffaudel, A., Daviaud, F., Dubrulle, B., Marié, L., Ravelet, F., Bourgoin, M., Odier, Ph., Pinton, J.-F. \& Volk, R. 2007 Magnetic field reversals in an experimental turbulent dynamo. Europhys. Lett. 77, 59001.

Chandrasekhar, S. 1961 Hydrodynamic and Hydromagnetic Stability. Clarendon.

Chiffaudel, A. \& Fauve, S. 1987 Strong resonance in forced oscillatory convection. Phys. Rev. A 35, 4004-4007.

Christensen, U. R., Olson, P. \& Glatzmaier, G. A. 1999 Numerical modelling of the geodynamo: a systematic parameter study. Geophys. J. Intl 138, 393-409.

Cortet, P.-P., Diribarne, P., Monchaux, R., Chiffaudel, A., Daviaud, F. \& Dubrulle, B. 2009 Normalized kinetic energy as a hydrodynamical global quantity for inhomogeneous anisotropic turbulence. Phys. Fluids 21, 025104.

Coullet, P. 1986 Commensurate-incommensurate transition in nonequilibrium systems. Phys. Rev. Lett. 56, 724-727.

Fuchs H., RÄDler K.-H. \& RheinhardT, M. 2001 Suicidal and parthenogenetic dynamos. In Dynamo and Dynamics, a Mathematical Challenge (ed. P. Chossat, D. Armbuster \& I. Oprea), pp. 339-347. Kluwer Academic.

Gailitis, A., Lielausis, O., Platacis, E., Dement’ev, S., Cifersons, A., Gerbeth, G., Gundrum, T., Stefani, F., Christen M. \& Will, G. 2001 Magnetic field saturation in the Riga dynamo experiment. Phys. Rev. Lett. 86, 3024-3027.

Gambaudo, J. M. 1985 Perturbation of a Hopf bifurcation by an external time-periodic forcing. $J$. Diff. Eq. 57, 172-199.

Giesecke, A., Nore, C., Plunian, F., Laguerre, R., Ribeiro, A., Stefani, F., Gerbeth, G., Léorat, J. \& Guermond, J.-L. $2009 a$ Generation of axisymmetric modes in cylindrical kinematic mean-field dynamos of VKS type. Geophys. Astrophys. Fluid Dyn. In press.

Giesecke, A., Stefani, F. \& Gerbeth, G. $2009 \mathrm{~b}$ Role of soft-iron impellers on the mode selection in the VKS dynamo experiment. Phys. Rev. Lett. Submitted.

Gissinger, C. 2009 A numerical model of the VKS experiment. Europhys. Lett. 87, 39002.

Guckenheimer, J. \& Holmes, P. 1986 Nonlinear Oscillations, Dynamical Systems and Bifurcations of Vector Fields. Springer.

Laguerre, R., Nore, C., Ribeiro, A., Léorat, J., Guermond, J.-L. \& Plunian, F. 2008 Impact of impellers on the axisymmetric magnetic mode in the VKS2 dynamo experiment. Phys. Rev. Lett. 101, 104501, 219902. 
Monchaux, R., Berhanu, M., Aumaître, S., Chiffaudel, A., Daviaud, F., Dubrulle, B., Ravelet, F., Fauve, S., Mordant, N., Pétrélis, F., Bourgoin, M., Odier, Ph., Pinton, J.-F., Plihon, N. \& VolK R. 2009 The von Kármán sodium experiment: turbulent dynamical dynamos. Phys. Fluids 21, 035108.

Monchaux, R., Berhanu, M., Bourgoin, M., Moulin, M., Odier, Ph., Pinton, J.-F., Volk, R., Fauve, S., Mordant, N., Pétrélis, F., Chiffaudel, A., Daviaud, F., Dubrulle, B., Gasquet, C. \& MARIÉ, L. 2007 Generation of a magnetic field by dynamo action in a turbulent flow of liquid sodium. Phys. Rev. Lett. 98, 044502.

Morin, V. 2005 Instabilités et bifurcations associées à la modélisation de la Géodynamo. PhD thesis, Universite Pierre et Marie Curie. Paris.

Morin, V. \& Dormy, E. 2009 Weak and strong field dynamos. Phys. Rev. Lett. Submitted.

Pétrélis, F. \& FAuve, S. 2008. Chaotic dynamics of the magnetic field generated by dynamo action in a turbulent flow. J. Phys. Condens. Matt. 20, 494203.

Pétrélis, F., Fauve, S., Dormy, E. \& Valet, J.-P. 2009 A simple mechanism for the reversals of Earth's magnetic field. Phys. Rev. Lett. 102, 144503.

Pétrélis, F., Mordant, N. \& Fauve, S. 2007 On the magnetic fields generated by experimental dynamos. Geophys. Astrophys. Fluid Dyn. 101, 289-323.

Ponty, Y., Laval, J.-P., Dubrulle, B., Daviaud, F. \& Pinton, J.-F. 2007 Subcritical dynamo bifurcation in the Taylor-Green flow. Phys. Rev. Lett. 99, 224501.

Ravelet, F., Berhanu, M., Monchaux, R., Bourgoin, M., Odier, Ph., Pinton, J.-F., Plihon, N., Volk, R., Fauve, S., Mordant, N., Pétrélis, F., Aumaître, S., Chiffaudel, A., Daviaud, F., Dubrulle, B. \& Marié, L. 2008 Chaotic dynamos generated by a turbulent flow of liquid sodium. Phys. Rev. Lett. 101, 074502.

Ravelet, F., Marié, L., Chiffaudel, A. \& Daviaud, F. 2004 Multistability and memory effect in a highly turbulent flow: experimental evidence for a global bifurcation. Phys. Rev. Lett. 93, 164501.

Rincon, F., Ogilvie, G. I. \& Proctor, M. R. E. 2007 Self-sustaining nonlinear dynamo process in Keplerian shear flows. Phys. Rev. Lett. 98, 254502.

RoberTs, P. H., 1978 Magneto-convection in a rapidly rotating fluid. In Rotating Fluids in Geophysics (ed. P. H. Roberts \& A. M. Soward), pp. 421-435. Academic.

Stieglitz, R. \& Müller, U. 2001 Experimental demonstration of a homogeneous two-scale dynamo. Phys. Fluids 13, 561-564. 